\title{
BROOKHTWEN
}

NATIONAL LABORATORY

BNL- 107211 -2014-CP

\section{Capacity Specification for Hybrid Energy Storage System to Accommodate Fast PV Fluctuations}

\author{
Xiaoyu Wang and Meng Yue \\ Presented at the 2015 IEEE PES General Meeting \\ Denver, Colorado \\ July 26-30, 2015 \\ December 2014 \\ Sustainable Energy Technologies Department \\ Brookhaven National Laboratory
}

\section{U.S. Department of Energy USDOE Office of Science}

Notice: This manuscript has been authored by employees of Brookhaven Science Associates, LLC under Contract No. DE-AC02-98CH10886 with the U.S. Department of Energy. The publisher by accepting the manuscript for publication acknowledges that the United States Government retains a non-exclusive, paid-up, irrevocable, world-wide license to publish or reproduce the published form of this manuscript, or allow others to do so, for United States Government purposes.

This preprint is intended for publication in a journal or proceedings. Since changes may be made before publication, it may not be cited or reproduced without the author's permission. 


\section{DISCLAIMER}

This report was prepared as an account of work sponsored by an agency of the United States Government. Neither the United States Government nor any agency thereof, nor any of their employees, nor any of their contractors, subcontractors, or their employees, makes any warranty, express or implied, or assumes any legal liability or responsibility for the accuracy, completeness, or any third party's use or the results of such use of any information, apparatus, product, or process disclosed, or represents that its use would not infringe privately owned rights. Reference herein to any specific commercial product, process, or service by trade name, trademark, manufacturer, or otherwise, does not necessarily constitute or imply its endorsement, recommendation, or favoring by the United States Government or any agency thereof or its contractors or subcontractors. The views and opinions of authors expressed herein do not necessarily state or reflect those of the United States Government or any agency thereof. 


\title{
Capacity Specification for Hybrid Energy Storage System to Accommodate Fast PV Fluctuations
}

\author{
Xiaoyu Wang \\ Meng Yue \\ Sustainable Energy Technologies Department \\ Brookhaven National Laboratory \\ Upton, NY 11973, NY, USA \\ \{xywang, yuemeng\}@bnl.gov
}

\begin{abstract}
To accommodate the fast power fluctuations associated with PV generation, a hybrid energy storage system (HESS) consisting of a battery energy storage system (BESS) and super-capacitor is evaluated in this paper. A probabilistic approach for determining the power capacity specification for the HESS is proposed. This method would allow the capacities of the BESS and super-capacitor to be properly designed to optimize the characteristics of high energy density of the BESS and high power density of the super-capacitor. Results show that the super-capacitor within the HESS helps to mitigate the high frequency fluctuations, which contributes to the extension of BESS lifetime. In addition, the super-capacitor helps to address the peaks in solar power fluctuations without the severe penalty of round trip losses associated with a BESS. The proposed approach has been simulated using real PV generation data from an existing PV power plant in Long Island, New York.
\end{abstract}

Index Terms--Battery energy storage system; hybrid energy storage system; non-parametric probability density estimation; super-capacitor; PV power fluctuation.

\section{INTRODUCTION}

With ever increasing concerns on energy security and environmental issues, utilization of renewable energy generation has gained rapid development and global acceptance over the past few decades. Among those sources, photovoltaic (PV) generation appears to be one of the most promising technologies due to the abundance of solar insolation in various regions, the absence of moving parts in the generation system, requiring no transmission lines (e.g. roof top PV installations), and low operation and maintenance costs [1] [2]. Unfortunately, renewable sources such as PV provide variable power generation. PV insolation striking on the PV panels is affected by environmental and cloud conditions, and it varies daily, hourly and even in seconds. As the output power of a PV plant fluctuates, this could cause the power grid to experience frequency and voltage deviations, especially if a large PV plant is connected to a weak grid. To mitigate the negative impacts of this variability from renewable power sources, the use of an energy storage system (ESS) has been considered [3-7]. Since different storage technologies have their own strengths and weaknesses in terms of cost, lifetime, and efficiency, a combination of different technologies, also called a hybrid energy storage system (HESS), has been proposed and evaluated to assist with the integration of renewable generation [8-15].

An HESS consisting of a BESS and a super-capacitor system is the main focus of this paper to accommodate the fast fluctuations within the PV power output. This combination was selected since they are both mature technologies. In [810], different control algorithms were proposed for the battery/super-capacitor hybrid storage systems. The authors focused mainly on the energy management system for the HESS in real time scale, while the capacity of the HESS was always assumed available to be managed. To achieve such an assumption in operation, appropriately sizing the HESS capacities is critical from an operation point of view, and this is generally carried out in the planning stage.

This paper is arranged as follows: the topology of the PV generation system investigated is described in Section II. The proposed power capacity specification method is described in Section III. A case study to verify the effectiveness of the proposed method is given in Section IV based on real PV data from an existing solar farm in Long Island, NY. Concluding remarks are given in the last section.

\section{TOPOLOGY DESCRIPTION}

The power conditioning system (PCS) for solar power plants may have different architectures-for example, a single-stage (grid-connected inverter-only) or a two-stage (a DC-DC converter and a grid-connected inverter) PCS [16, 17]. The latter structure, shown in Fig. 1, is more popular and was selected to interface a PV power plant with a transmission network because it may provide an extra degree of freedom of control. In this topology, the PV-connected DC-DC converter functions to continuously track the maximum power point (MPP) of the solar power plant by regulating the PV modules' 
output voltage, generating $\mathrm{PV}$ power, $P_{P V}$. The grid-interfaced inverter is controlled to convert the $\mathrm{DC}$ power to AC format and regulate the real and reactive power flowing into the grid instantaneously and independently. It is assumed that the power factor of the inverter is kept at unity and the delivered power to grid is $P_{d}$.

In the PCS system described above, a HESS is connected to the dc-link for either storing the excess energy generated by the PV plant or releasing energy to support the grid when the generated solar power is deficient. This charging/discharging process is managed by the control system devised for the HESS, which is out of scope of this paper and one assumes the control scheme can properly regulate the power outputs from BESS and super-capacitor within the HESS.

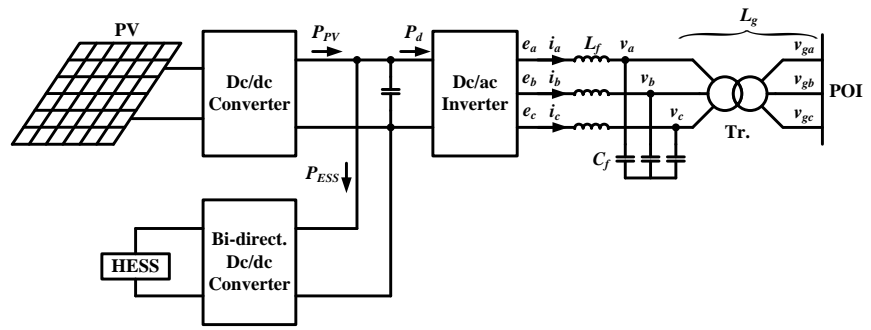

Fig. 1. Overall system diagram of a PV system incorporating HESS.

\section{HESS POWER CAPACITY SPECIFICATION}

\section{A. Analysis on Solar Power Profiles}

To study the capacity specification of the HESS in smoothing PV power fluctuation, a time series PV power data profile is assumed known. A typical PV output profile on a day during fall 2014 in Northeast region is shown in Fig. 2.

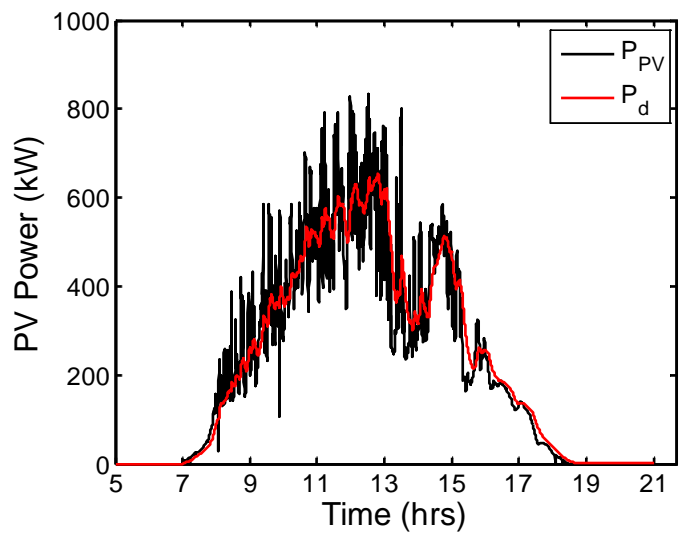

Fig.2. A typical PV power profile based on data taken from Long Island Solar Farm (LISF): smoothed $P_{d}$ is shown.

As shown in Fig. 2, the dispatched level of the PV farm is assumed to be $P_{d}$, i.e., a smoothed out power, $P_{d}$ is expected to be dispatched from the solar farm to the grid. It is noted that the reduced fluctuations with $P_{d}$ will cause less negative impacts on the system operations, such as frequency and voltage deviations. The HESS is designed to accommodate the difference between $P_{P V}$ and $P_{d}$. In this paper, $P_{d}$ is obtained by the following equation,

$$
P_{d}(s)=P_{P V}(s) /\left(1+T_{1} s\right)
$$

where, $s$ is the Laplace operator, and time constant $T_{1}$ is the smoothing factor, which value determines the smoothness of the $P_{d}$ profile. In this paper, $T_{1}$ is selected to be $1 \mathrm{~min}$. for illustration purpose. In real world, it can be quantified by the operation requirements, such as the maximum ramp rates from the dispatched output from PV plant. This will be further investigated and reported in future work.

The power profile of the HESS, $P_{E S S}(t)$ can be derived from,

$$
P_{E S S}(t)=P_{P V}(t)-P_{d}
$$

From (2), for a given $P_{P V}, P_{E S S}(t)$ will vary according to the $T_{1}$ values. For example, for the $P_{P V}$ profile given in Fig. 2, the $P_{E S S}(t)$ profile given in Fig. 3 corresponds to $T_{1}=1 \mathrm{~min}$., indicated by the red line in Fig. 2.

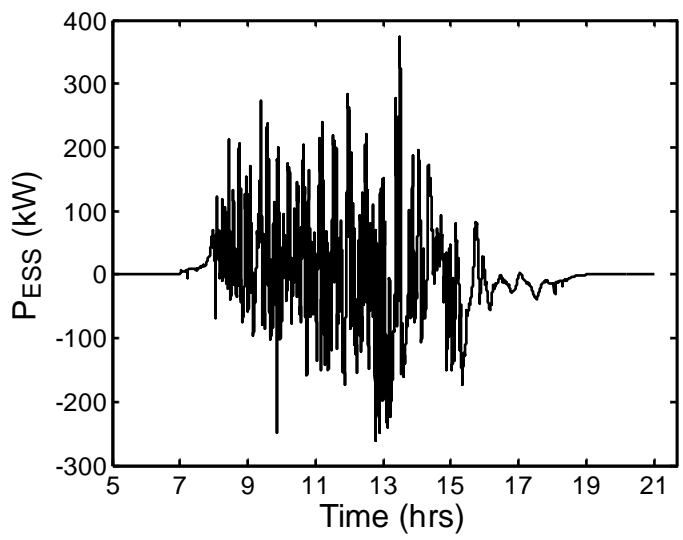

Fig. 3. HESS power profile for $T_{1}=1 \mathrm{~min}$.

With reference to Fig. 3, the positive values indicate that the HESS is charged to store the surplus energy produced from the solar farm while the negative values show that the HESS releases power to maintain the dispatched power profile. For the purpose of sizing the HESS power capacity, however, the HESS must be sized to the maximum expected power flow to satisfy the system design requirements. Therefore, we can use the absolute value of power fluctuations, $\left|P_{E S S}\right|$, as shown in Fig. 4.

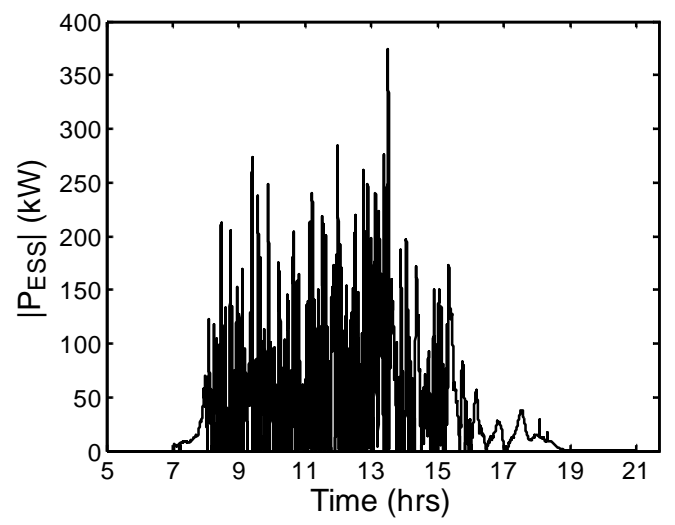

Fig. 4. Absolute values of HESS power profile for $T_{1}=1 \mathrm{~min}$.

To extend the life length of a BESS, it is best to avoid repeated charging/discharging at the peak/valley values, since 
the high charge/discharge currents are detrimental to the battery system. Considering its high power density capability, a super-capacitor system can be assigned to accommodate the peak/valley values. Hence, its BESS counterpart can handle the low fluctuating parts of the PV power, which improves its life span. Another advantage for this combination is that the high energy capacity of the BESS complements the drawback of less energy capacity of the super-capacitor. The following contents elaborate how to assign the power ratings of the BESS and super-capacitor in the HESS. Here, the HESS profile in Fig. 4 is interpreted using a histogram, as shown in Fig. 5, from which it can be easily seen that frequency of the peak values in the HESS power profile is very low, i.e. the peak values occur very rarely compared to the lower values, say $20 \mathrm{~kW}$.

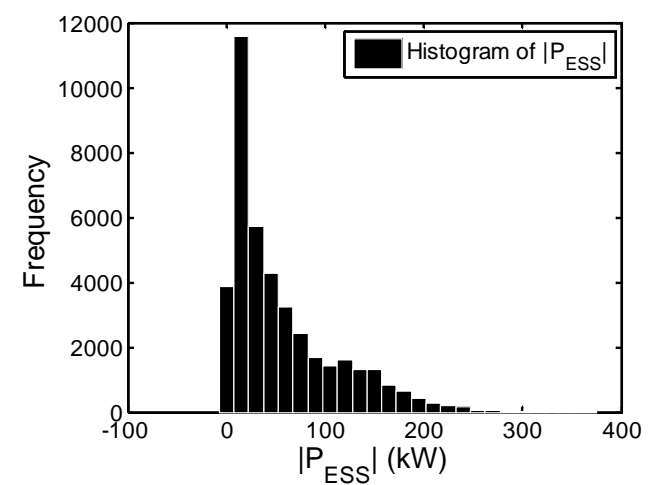

Fig. 5. Histogram of the absolute values of HESS power profile for $T_{1}=1$ min.

To get a clearer view of the distribution of the power profile shown in Fig. 5, different probability density functions (PDFs) can be selected to fit the histogram in Fig. 5. Compared with the parametric methods, for example Weibull or Rayleigh distributions, the non-parametric PDFs possess the advantage that they can reflect the practical data clearly [18]. A non-parametric PDF estimation method, Kernel smoothing density estimation, is adopted in this paper, as introduced in the next section.

\section{B. Kernel Smoothing Density Estimation and its Application to Size HESS}

In statistics, kernel density estimation (KDE) is a nonparametric way to estimate the probability density function of a random variable. Let $\left(x_{1}, x_{2}, \ldots, x_{n}\right)$ be an independent and identically distributed random sample drawn from some distribution with an unknown density $\Phi$. Its kernel density estimator is,

$$
\Phi_{\text {ess }, h}(x)=\frac{1}{n} \sum_{i=1}^{n} K_{h}\left(x-x_{i}\right)=\frac{1}{n h} \sum_{i=1}^{n} K\left(\frac{x-x_{i}}{h}\right)
$$

where $K(\bullet)$ is the kernel function, a symmetric but not necessarily positive function that integrates to one. $h>0$ is a smoothing parameter called the bandwidth. A kernel with subscript $h$ is called the scaled kernel and defined as $K_{h}(x)=$ $1 / h K(x / h)$. Intuitively, $h$ can be chosen as small as the data allow, and one normally selects a value equal to the $100^{\text {th }}$ of the data range $\left[x_{1}, x_{n}\right]$.

It is noted that a range of kernel functions are commonly used. In this paper the normal kernel function is adopted to calculate the probability density estimation of $\left|P_{E S S}\right|$ based on the histogram given in Fig. 5, by using the Kernel Smoothing Density Estimation (KSDE) function provided in the Matlab Statistic Toolbox, i.e., the function,

$$
\left[\Phi_{\text {est }},\left|P_{E S S}\right|, i\right]=\operatorname{KSDE}\left(\left|P_{E S S}\right|\right)
$$

returns the vector of density values, $\Phi_{\text {est }}$, evaluated at the points in $\left|P_{E S S}\right|, i$. By default, the density is evaluated at 100 equally spaced points that cover the range of the data in $\left|P_{E S S}\right|$, which is theoretically optimal for estimating densities for the normal distribution [19].

By applying KSDE to the histogram shown in Fig. 5, the PDF of the absolute values of HESS power, $\Phi_{\text {est }}\left(\left|P_{E S S}\right|\right)$ is obtained as shown in Fig. 6.

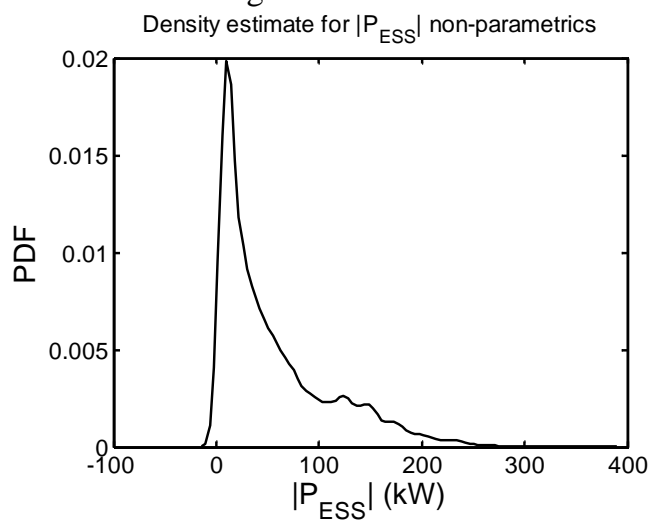

Fig. 6. Estimated density function of $\left|P_{E S S}\right|$ for $T_{1}=1$ min.

C. Probabilistically Sizing the BESS and the Supercapacitor

Based on the PDF given in Fig. 6, the cumulative density function (CDF) of the absolute values of HESS power profile $F(x)$, is readily obtained as,

$$
F(x)=\int_{0}^{x} \Phi_{\text {est }}\left(\left|P_{E S S}\right|\right) d\left|P_{\text {ESS }}\right|
$$

The CDF obtained from (4) is shown in Fig. 7. From the curve shown in Fig. 7 and the analysis in Section III.A, one can specify the BESS capacity to accommodate a given confidence level, say $97.5 \%$ of the required HESS power in Fig. 4, which corresponds to the point shown in Fig. 7 at $P_{b, T_{1}=1 \min }=197.38 \mathrm{~kW}$ for $\mathrm{CDF}=0.975$, or,

$$
P_{b, T_{1}=1 \min }=F^{-1}(0.975)
$$

The remaining $2.5 \%$ of mismatch power in Fig. 4 is supplied by the super-capacitor within HESS. Therefore, the power capacity of the super-capacitor, $P_{C, T_{1}=1 \min }$ can be specified by,

$$
P_{C, T_{1}=1 \min }=\max \left(\left|P_{E S S}\right|\right)-P_{b, T_{1}=1 \min }
$$

\section{ILLUSTRATION}

The PV power data obtained from an existing solar farm is used to illustrate the effectiveness of the HESS design procedure proposed in Section III. In the study, PV power 
profile of a fall day (September 24, 2014) was obtained from the solar farm at Long Island, U.S. Although in a real application it requires a longer period of observation than twenty four hours, for the purpose of illustration, we only use the 24 hours duration. The PV power data was then averaged over 10-second intervals, as shown in Fig. 2. It is noted the HESS model developed in Matlab/Simulink platform was adopted in this case study, details can be found in [20].

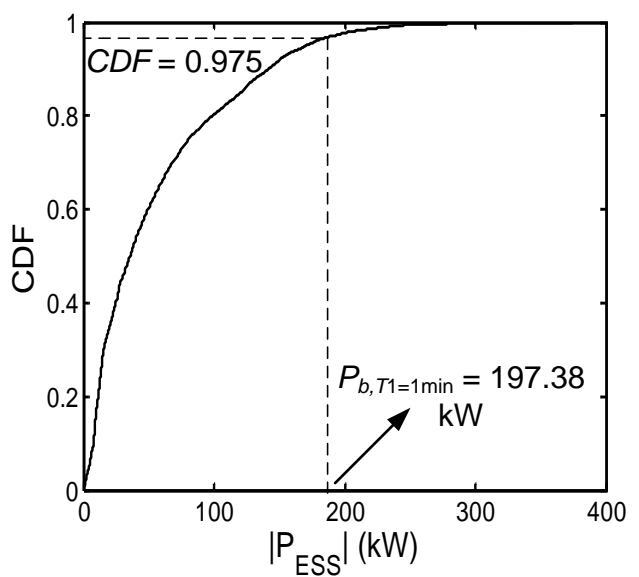

Fig. 7. CDF of $\left|P_{E S S}\right|$ for $T_{1}=1 \mathrm{~min}$.

Simulation results for the battery model are shown in Fig. 8. In this simulation, the battery system is specified as follows. Under normal condition, the dc-link voltage of the back-to-back converter is assumed to be $600 \mathrm{~V}$, while the terminal voltage of the Hawker Genesis 12V26Ah10EP sealed valve-regulated lead-acid (VRLA) is $12.96 \mathrm{~V}$ at $S O C=$ 1. Therefore, to build up the required dc-link voltage, one can connect the battery units in series. The series connected battery units can be calculated to be $600 / 12.96$ or 47 . The capacity of one battery unit available is $26 \mathrm{Ah}$, and hence the power capacity for each series connected branch is $(26 / 5) * 609.12=3.17 \mathrm{~kW}$. To accommodate the peak value of the ESS variations, the parallel connected branch number of the battery system can be specified to be $374.8 \mathrm{~kW} / 3.17 \mathrm{~kW}$ $=119$. The terminal voltage profile over the investigated day is given in Fig. 8(a). Fig. 8(b) shows the battery current profile.

For comparison, in the second simulation, the HESS consisting of battery storage and super-capacitor is evaluated. In this HESS configuration, the Hawker Genesis 12V26Ah10EP batteries and Nesscap 2.7V/600F supercapacitors within the HESS are specified as follows. From probabilistic analysis, the battery system capacity is 197.38 $\mathrm{kW}$ and the super-capacitor capacity is $177.42 \mathrm{~kW}$. For the battery system, a similar specification method to that used in last simulation is deployed and it is specified to be 47 units connected in series and 63 branches connected in parallel.

For the super-capacitor system, the rated voltage of the super-capacitor is $2.7 \mathrm{~V}$ and it needs to connect 223 units in series to build up the normal dc-link voltage of $602.1 \mathrm{~V}$. From the datasheet of Nesscap 2.7V/600F, one can find that the rated current of the super-capacitor unit is 145 A. Therefore, it can be readily derived that the rated power of each super-capacitor string is $87.3 \mathrm{~kW}$. Therefore, it needs to connect $177.42 / 87.3$ or about 3 strings in parallel.

The HESS as specified above is used to accommodate the ESS power profile shown in Fig. 3. The power profile is fed to the HESS input and then divided by the terminal voltage to obtain the HESS input current. The procedure described in [20] is adopted to calculate the battery and super-capacitor currents based on the current pulses. Simulation results are shown in Fig. 9.

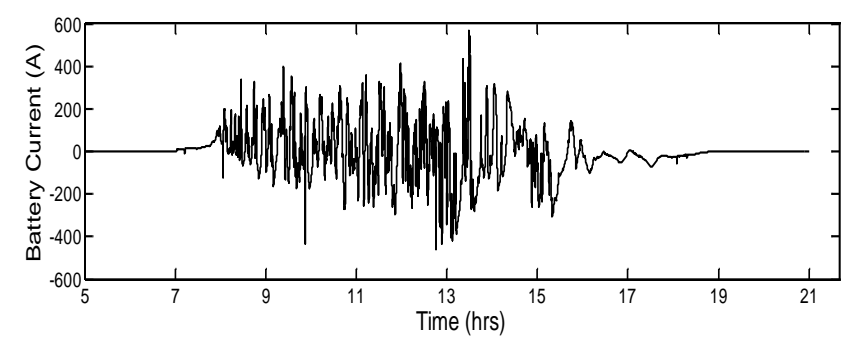

(a)

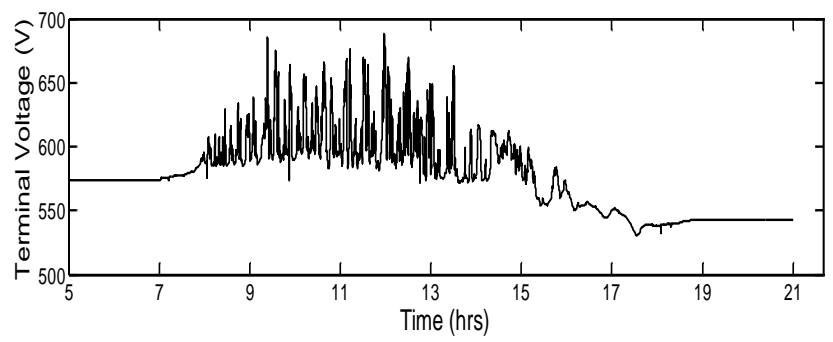

(b)

Fig. 8. Simulation results of the battery model for the solar power fluctuation smoothing, profiles of (a) battery terminal voltage, (b) battery current.

The HESS current profile shown in Fig. 9(a) is similar with that shown in Fig. 8(b). The current flowing through the super-capacitors within the HESS is given in Fig. 9(b). It can be observed from this figure that the high frequency fluctuations appearing in Fig. 9(a) are accommodated mostly by the super-capacitors. The rest of the current with low fluctuations is taken by the battery units within the HESS, as shown in Fig. 9(c). This lower frequency fluctuation will affect the life extension of the battery pack. The terminal voltage of the HESS is shown in Fig. 9(d), which has fewer fluctuations compared with that given in Fig. 8(a), where only BESS alone is used.

\section{CONCLUSIONS}

To accommodate fast solar power fluctuations due to the intermittent nature of irradiance conditions, a hybrid energy storage system (HESS) consisting of a traditional battery energy storage system (BESS) and a super-capacitor is evaluated in this study.

A probabilistic power capacity specification method for the BESS and super-capacitor within the HESS is proposed in this paper. The BESS capacity is specified to handle the solar power fluctuation with a magnitude less than a certain value corresponding to a preset confidence level on the cumulative density function (CDF) of $\left|P_{E S S}\right|$. The remaining part of the 
HESS power capacity with high magnitude fluctuations is assigned to the super-capacitor. In this way, the supercapacitor can take over the peaks/valleys of the solar power due to its high power density property, which is beneficial for BESS life.

Illustration of the above proposed scheme has been shown in this paper and the simulation results verified the effectiveness of this method in HESS power capacity specification. While in this paper, we specify the power capacities for a given smooth factor, $T_{1}$, future work will investigate how this value impacts the HESS capacities and how to choose the optimal value to achieve best economic benefit.

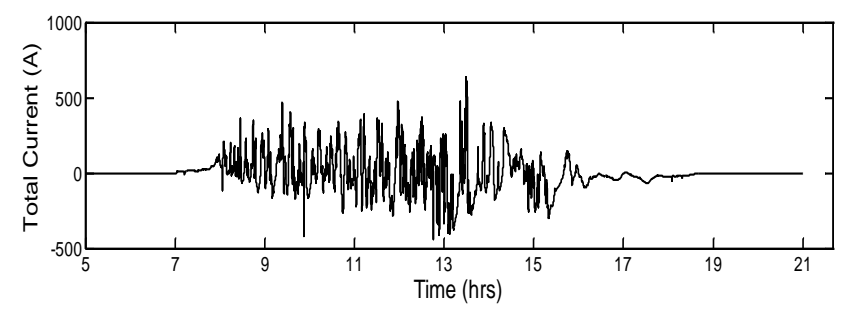

(a)

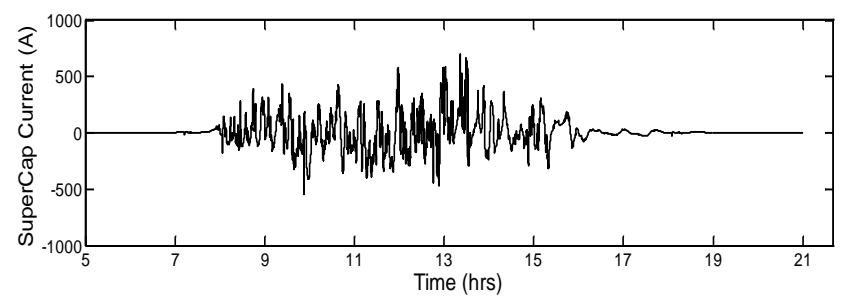

(b)

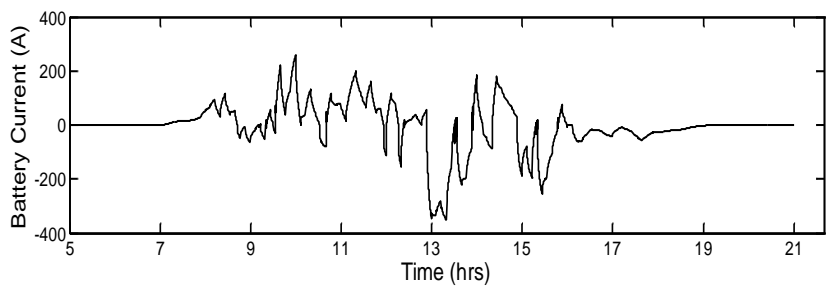

(c)

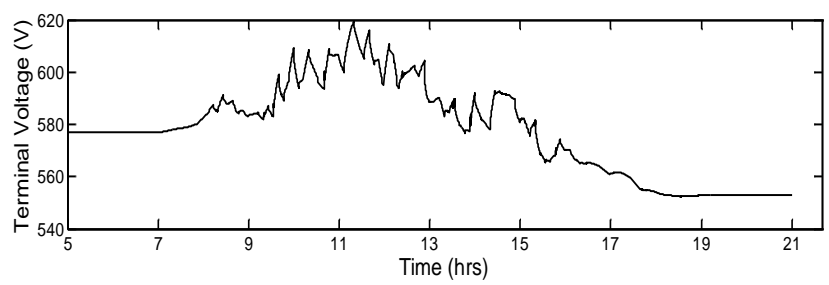

(d)

Fig. 9. Simulation results of the HESS model for the solar power fluctuation smoothing, profiles of (a) the total charge/discharge current, (b) supercapacitor current, (c) battery current, (d) HESS terminal voltage.

\section{REFERENCES}

[1] "Trends in Photovoltaic Applications," IEA Report IEA-PVPS T120:2011, 2011.

[2] S. Achilles, S. Schramm, J. Bebic, G. G. Research, and N. R. E. Laboratory, Transmission system performance analysis for highpenetration photovoltaics: National Renewable Energy Laboratory, 2008.
[3] Z. A. Styczynski, P. Lombardi, R. Seethapathy, M. Piekutowski, C. Ohler, B. Roberts, et al., "Electric Energy Storage and its tasks in the integration of wide-scale renewable resources," in Integration of WideScale Renewable Resources Into the Power Delivery System, 2009 CIGRE/IEEE PES Joint Symposium, 2009, pp. 1-11.

[4] S. C. Smith, P. K. Sen, and B. Kroposki, "Advancement of energy storage devices and applications in electrical power system," in Power and Energy Society General Meeting - Conversion and Delivery of Electrical Energy in the 21st Century, 2008 IEEE, 2008, pp. 1-8.

[5] C. Abbey and G. Joos, "A Stochastic Optimization Approach to Rating of Energy Storage Systems in Wind-Diesel Isolated Grids," Power Systems, IEEE Transactions on, vol. 24, pp. 418-426, 2009.

[6] C. Abbey, K. Strunz, and G. Joos, "A Knowledge-Based Approach for Control of Two-Level Energy Storage for Wind Energy Systems," Energy Conversion, IEEE Transactions on, vol. 24, pp. 539-547, 2009.

[7] A. Esmaili and A. Nasiri, "Energy storage for short-term and long-term wind energy support," in IECON 2010 - 36th Annual Conference on IEEE Industrial Electronics Society, 2010, pp. 3281-3286.

[8] F. S. Garcia, A. A. Ferreira, and J. A. Pomilio, "Control Strategy for Battery-Ultracapacitor Hybrid Energy Storage System," in Applied Power Electronics Conference and Exposition, 2009. APEC 2009. Twenty-Fourth Annual IEEE, 2009, pp. 826-832.

[9] L. Wei, G. Joos, and J. Belanger, "Real-Time Simulation of a Wind Turbine Generator Coupled With a Battery Supercapacitor Energy Storage System," Industrial Electronics, IEEE Transactions on, vol. 57, pp. 1137-1145, 2010.

[10] H. Jia, Y. Fu, Y. Zhang, and W. He, "Design of Hybrid Energy Storage Control System for Wind Farms Based on Flow Battery and Electric Double-Layer Capacitor," in Power and Energy Engineering Conference (APPEEC), 2010 Asia-Pacific, 2010, pp. 1-6.

[11] K. Ogimi, A. Yoza, A. Yona, T. Senjyu, and T. Funabashi, "A study on optimum capacity of battery energy storage system for wind farm operation with wind power forecast data," in Harmonics and Quality of Power (ICHQP), 2012 IEEE 15th International Conference on, 2012, pp. 118-123.

[12] A. Uehara, T. Senjyu, Y. Kikunaga, A. Yona, N. Urasaki, T. Funabashi, et al., "Study on optimum operation planning of wind farm/battery system using forecasted power data," in Power Electronics and Drive Systems, 2009. PEDS 2009. International Conference on, 2009, pp. 907-912.

[13] R. Watanabe, Y. Ito, Y. Hida, R. Yokoyama, K. Iba, and T. Tsukada, "Optimal capacity selection of hybrid energy storage systems for suppressing PV output fluctuation," in Innovative Smart Grid Technologies - Asia (ISGT Asia), 2012 IEEE, 2012, pp. 1-5.

[14] K. W. Wee, S. S. Choi, and D. M. Vilathgamuwa, "Design of a renewable - hybrid energy storage power scheme for short-term power dispatch," in Electric Utility Deregulation and Restructuring and Power Technologies (DRPT), 2011 4th International Conference on, 2011, pp. 1511-1516.

[15] X. Y. Wang, D. Mahinda Vilathgamuwa, and S. S. Choi, "Determination of Battery Storage Capacity in Energy Buffer for Wind Farm," Energy Conversion, IEEE Transactions on, vol. 23, pp. 868878, 2008.

[16] W. Kramer, S. Chakraborty, B. Kroposki, and H. Thomas, "Advanced Power Electronic Interfaces for Distributed Energy Systems, Park 1: Systems and Topologies," Technical Report, NREL/TP-581-42672, March, 2008.

[17] F. Schimpf and L. E. Norum, "Grid Connected Converters for Photovoltaic, State of the Art, Ideas for Improvement of Transfomerless Inverters," Nordic Workshop on Power and Industrial Electronics, June, 2008.

[18] D. S. Callaway, "Sequential Reliability Forecasting for Wind Energy: Temperature Dependence and Probability Distributions," Energy Conversion, IEEE Transactions on, vol. 25, pp. 577-585, 2010.

[19] A. W. Bowman and A. Azzalini, Applied Smoothing Techniques for Data Analysis. NY: Oxford University Press, 1997.

[20] X. Y. Wang, M. Yue, E. Muljadi, and W. Gao, "Probabilistic Approach for Power Capacity Specification of Wind Energy Storage Systems," Industry Applications, IEEE Transactions on, vol. 50, pp. 1215-1224, 2014. 